\title{
A case of fulminant pneumococcus meningoencephalitis progressing with white matter involvement despite two doses of conjugated pneumococcus vaccine
}

\author{
Ebru Azapağası ${ }^{1 \oplus}$, Tanıl Kendirli ${ }^{1 \odot}$, Gökçen Öz Tuncer ${ }^{2 \odot}$, Serhan Özcan ${ }^{1 \oplus,}$ \\ Halil Özdemir ${ }^{3 \odot}$, Suat Fitöz ${ }^{4 \oplus}$, Erdal İnce ${ }^{3 \odot}$ \\ Departments of ${ }^{1}$ Pediatric Intensive Care, ${ }^{2}$ Pediatric Neurology, ${ }^{3}$ Pediatric Infection and ${ }^{4}$ Pediatric Radiology, Ankara University \\ Faculty of Medicine, Ankara, Turkey.
}

\begin{abstract}
Background. Streptococcus pneumonia is a cause of serious mortality and morbidity, especially among small children. However, currently, it causes lower rates of invasive infections due to successful vaccination programs.

Case. We report an exceptional case of a 6-month-old child with meningoencephalitis caused by Streptococcus pneumonia despite the administration of two doses of pneumococcal conjugate vaccine (PCV). Meningitis progressed rapidly and led to widespread damage in parenchymal brain tissue with the emergence of fulminant meningoencephalitis. Computed tomography (CT) and magnetic resonance imaging (MRI) revealed widespread brain lesions, suggesting extensive parenchymal injury.

Conclusion. Such diffuse white matter lesions among pediatric patients with Streptococcus pneumonia meningoencephalitis despite two doses of PCV have not been reported previously.
\end{abstract}

Key words: pneumococcal conjugate vaccine, meningoencephalitis, meningitis, streptococcus pneumoniae, serotype $19 \mathrm{~F}$.

Streptococcus pneumoniae is the leading cause of community-acquired invasive bacterial infections in young children and is a cause of serious mortality and morbidity. ${ }^{1}$ Pneumococcal conjugate vaccines $(\mathrm{PCV})$ were developed over the last decade to provide effective vaccine protection, particularly in young children. Conjugate vaccines were demonstrated to be effective for reducing transmission and protecting against invasive disease., ${ }^{2,3}$

Complications linked to due S. pneumoniae infection occur due to the invasive and immunogenic effects of alpha-hemolytic streptococci. Pneumococcal meningitis causes cerebral vasculitis. ${ }^{4}$

$凶 \quad$ Ebru Azapagasi

ebruazapagasi@hotmail.com

Received 11st October 2017, revised 19th May 2020,

30th July 2020, accepted 10th August 2020.
When both severe meningeal and encephalitic findings are present, the term meningoencephalitis may be used. ${ }^{5}$ However, the extension of $\mathrm{S}$. pneumonia infection to the parenchyma of the central nervous system (CNS) in adults and children has only rarely been reported..$^{5-7}$

In this report, we present a 6-month-old infant who developed. S. pneumoniae meningitis despite the administration of two doses of PCV and whose meningitis progressed rapidly and led to widespread damage in parenchymal brain tissue with the emergence of fulminant meningoencephalitis.

\section{Case Report}

A 6-month-old male patient was referred to the emergency clinic of our hospital with symptoms of high fever, alteration in consciousness, ptosis 
of the left eyelid and seizure. It was learned from his history that he had no previous problems. He had projectile vomiting and a high fever for several days. One day before attendance at the hospital, he had changes of consciousness and loss of appetite.

It was also learned that he was the second child of parents who were not relatives and was fed with breastmilk within the first four months and then supplements were added. His neurologic development was appropriate for age. Two doses of pneumococcal conjugate vaccines had been administered.

When he was referred to the pediatric emergency clinic, his general condition was poor and he was unconscious.

His rectally measured body temperature was $39.7^{\circ} \mathrm{C}$, heart rate 125 beats $/ \mathrm{min}$, blood pressure $80 / 55 \mathrm{~mm} \mathrm{Hg}$ and oxygen saturation $96 \%$.

On neurologic examination, anisocoria was present. There was ptosis in the left eye. A light reflex could not be elicited in the left eye. In the right eye, the direct light reflex was positive. The deep tendon reflex could be elicited and foot sole skin reflexes were bilaterally positive. Four extremities were spontaneously mobile, there was no clear lateralizing muscle strength deficit. The Glasgow Coma Score was 7. The child was intubated and accepted to our pediatric intensive care unit (PICU).

Blood count results performed after the patient was admitted were as follows: Hemoglobin: $12 \mathrm{~g} / \mathrm{dL}$, white blood cell (WBC): 7800/ $\mathrm{mm}^{3}$, thrombocyte: $347,000 / \mathrm{mm}^{3}$, C-reactive protein:150 mg/L, PTT/PT:48/24 seconds, and INR: 2.1. Biochemical parameters were found to be normal. Blood and urine cultures were obtained. Viral and bacterial respiratory tract samples were sent for polymerase chain reaction (PCR) analysis. Contrast-enhanced brain computed tomography (CT) was normal.

In a lumbar puncture (LP), Gram staining revealed abundant Gram-positive cocci in pairs. (Fig. 1). Cerebrospinal fluid (CSF) examination revealed a turbid fluid with a WBC count of 250/ $\mathrm{mm}^{3}$ (100\% polymorphonuclear cells), protein $420 \mathrm{mg} / \mathrm{dl}$ (reference value: 15-45 mg/dl), and glucose $1 \mathrm{mg} / \mathrm{dl}$. Simultaneous blood glucose was $178 \mathrm{mg} / \mathrm{dl}$. Gram-positive diplococcus was seen on a Gram-stained preparation of the CSF.

Acute bacterial meningitis was diagnosed and treatment with intravenous ceftriaxone (100 mg/kg/day), vancomycin (60 mg/kg/day), and dexamethasone $(0.15 \mathrm{mg} / \mathrm{kg}$ every $6 \mathrm{~h})$ was initiated. In follow-up, there was a clonic seizure in the right arm lasting for 15 seconds; phenytoin was started. Within the first day, both blood culture and cerebrospinal fluid yielded a pure growth of S. Pneumoniae, type 19 F. Rifampicin was added to treatment. On a neurologic examination performed one day later, dystonia was detected and clonazepam was initiated. No evidence was found for other (viral) causes of meningitis/encephalitis.

With no improvement to the general status on monitoring, cranial magnetic resonance imaging (MRI) was performed. Ischemic areas were observed in basal ganglions, left dentate nucleus, external capsules, medial lemniscus, corpus callosum splenium, temporal lobes, and the centrum semiovale, showing widespread

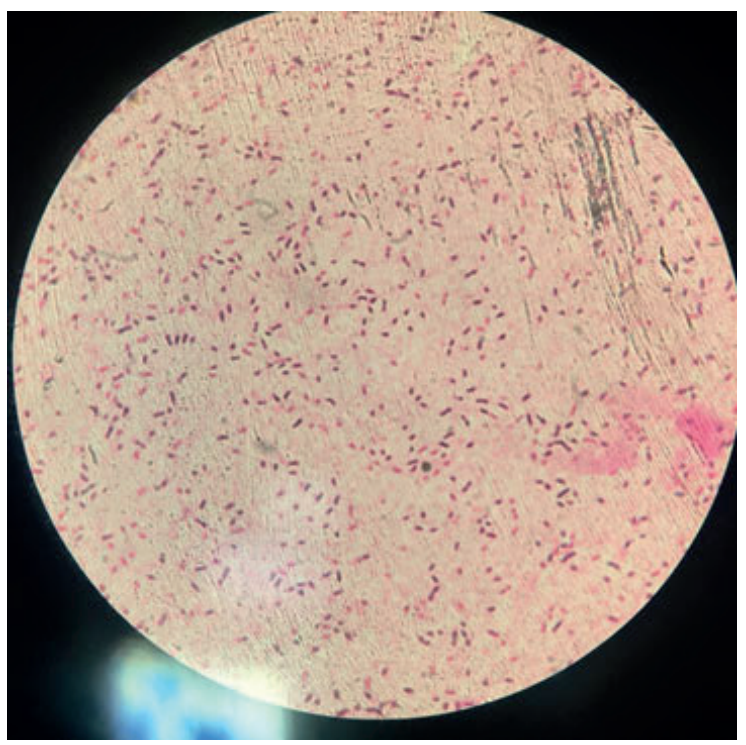

Fig. 1. Diplococci were commonly observed with gram staining of CSF. 

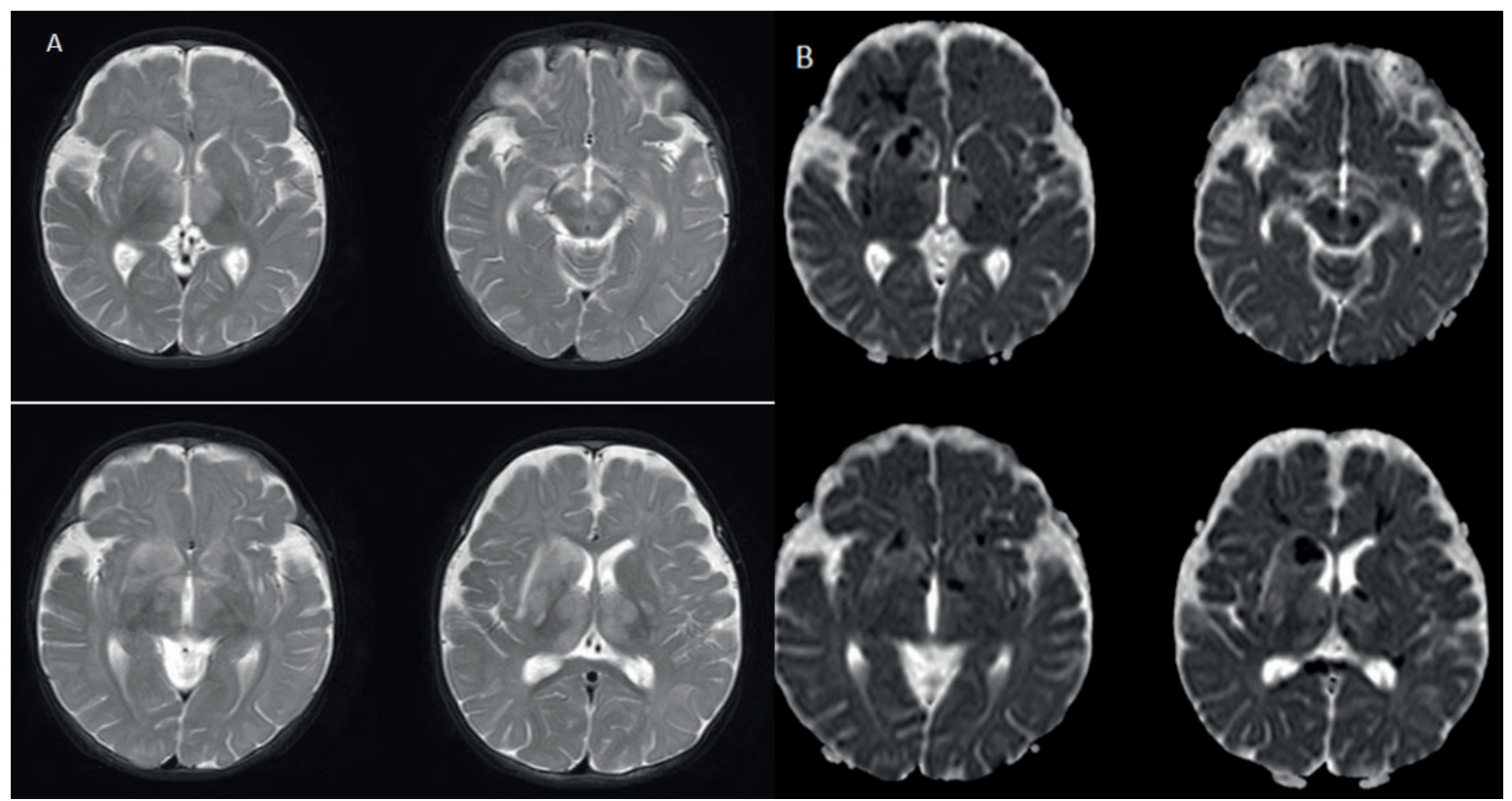

Fig. 2A. T2-weighted cranial magnetic resonance images show hyperintense pathologic signal changes in bilateral lentiform and caudate nucleus as well as in bilateral thalamus, red nucleus, lemniscusmedialis and external capsule. 2B. Corresponding ADC (Apparent Diffusion Coefficient) maps demonstrate restricted diffusion in hyperintense areas suggesting acute ischemic foci.

diffusion restriction on T2-weighted images (Fig. 2A and B). Diffusion-weighted (DW) MRI was suggestive of meningovasculitis with multiple bilateral small infarcts, possibly secondary to small-vessel vasculitis.

On a follow-up CT 6 days later, hypodense areas were observed in the left dentate nucleus, pons posterior and mesencephalon, thalamus anteromedial and bilateral basal ganglions, external capsule, internal capsule, and the level of the centrum semiovale. Findings were evaluated as secondary to meningoencephalitis. Also, expansion was observed in the ventricles and hydrocephalus had developed. Daily head circumference measurements were planned.

Minimal inhibitory concentration (MIC) levels of ceftriaxone were $0.38 \mu \mathrm{g} / \mathrm{mL}$. Rifampicin and vancomycin were stopped. Ceftriaxone was continued. The respiratory tract was negative for viral PCR. Levetiracetam was added to the treatment because of his seizures.

On the 12th day of admission to the PICU, the boy's head circumference increased and cranial tomography was performed again. Occasional pathological contrast involvement considered secondary to a linear infarct was observed on the right at the level of the putamen and thalamus. Hypodense areas were observed on the right in both front regions secondary to infarctus in a broad area or linked to cerebritis in the periventricular white matter.

A drain was inserted on the $13^{\text {th }}$ day due to increased hydrocephalus (Fig. 3). Extubation was finally achieved on day 15 after admission, and on day 18 the patient was transferred to the pediatric infectious disease ward.

There was no growth in a follow-up CSF culture. External drainage was removed on the $26^{\text {th }}$ day and a V-P shunt was inserted.

In visual evoked potential tests, the patient had a latency of $195 \mathrm{msec}$ on the right and 122 msec on the left, with clear extension found. Brainstem auditory evoked response identified bilateral sensorineural hearing loss. No immune deficiency was detected in the patient. The patient was discharged to the pediatric infection 


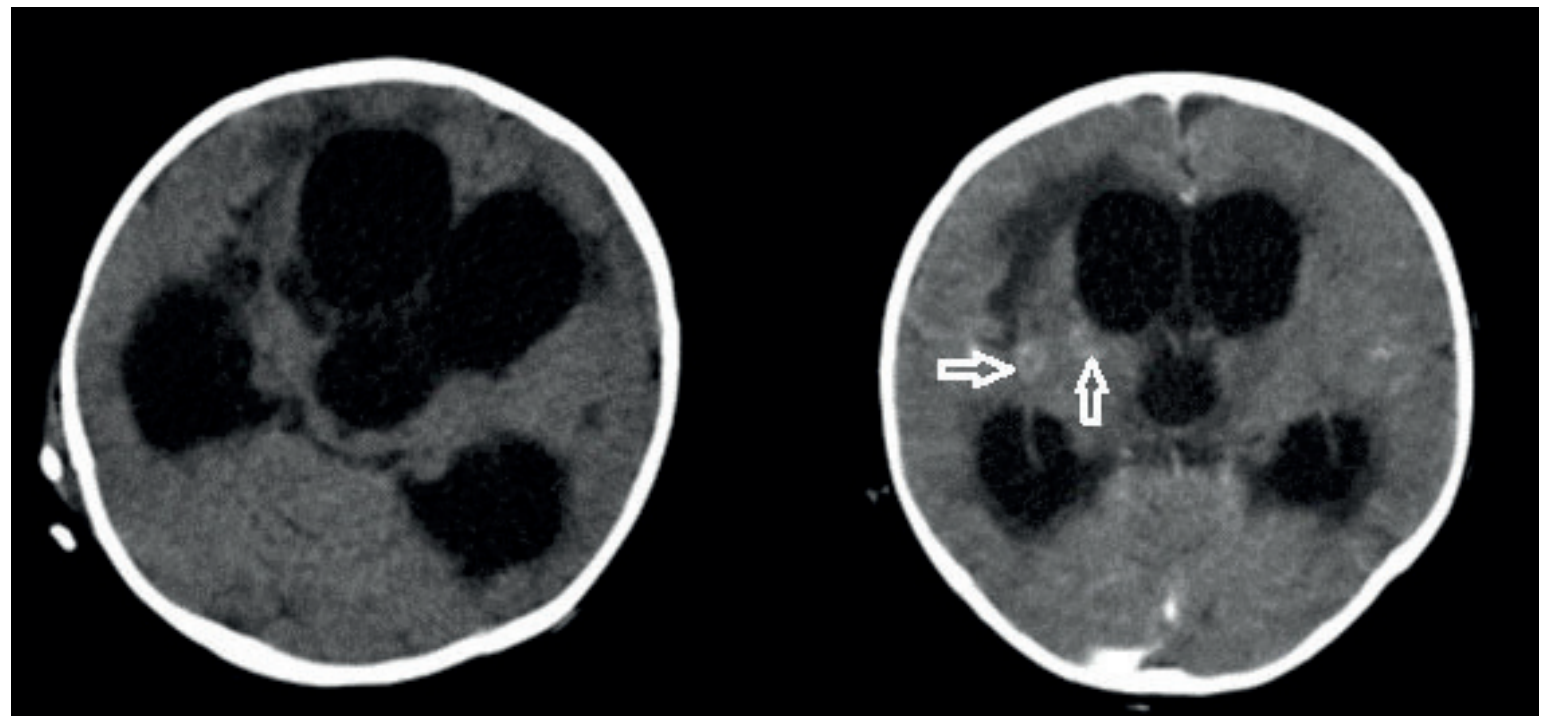

Fig. 3. Control cranial computed tomography.

Dilatation in bilateral lateral ventricle and 3rd ventricle; secondary hypodense areas of chronic infarctus in bilateral frontal periventricular white matter, more pronounced on the right; and hypodense area linked to resorption of transependymal cerebrospinal fluid in the bilateral periventricular region.

ward on the $28^{\text {th }}$ day of hospital admission with levetiracetam, phenytoin, and clonazepam drops.

The patient is currently aged 4 years and continues to attend the rehabilitation center. His mental and motor development is poor, and he has anisocoria and mydriasis in the left eye.

Written consent for publication of this case report and accompanying images was obtained from the parents of the patient.

\section{Discussion}

S. Pnemoniae meningitis is an important cause of morbidity and mortality in young children worldwide. ${ }^{8}$ Neurologic complications include seizures, diffuse brain edema, hydrocephalus, hearing loss and ischemic or hemorrhagic brain damage. ${ }^{7}$ Cerebral vasculopathy is one of the major complications of bacterial meningitis. ${ }^{9}$ In a study of pediatric patients by Synder et al., ${ }^{10}$ the rate of cerebrovascular complications associated with bacterial meningitis was reported to be $30 \%$. In a retrospective study of 87 adult cases of pneumococcal meningitis, the incidence of arterial cerebrovascular complications was reported to be as high as $21.8 \% .^{5}$ The agent that causes these complications is most frequently $S$. Pneumoniae. ${ }^{9,11}$

Thirteen valent pneumococcal conjugate vaccines (PCV13) were integrated into the expanded immunization program in Turkey in $2011 .^{12}$ The serotypes in the vaccines were 4,6B,9V,14,18C,19F,23F, 1,5,7F,3,6A, and 19A. ${ }^{11}$ PCV13, transported under cold chain conditions under supervision within the framework of the Ministry of Health regulations is administered to children at the ages of 2-4-6, and 18 months. The present patient was administered two doses of PCV13 per the routine vaccine protocol. Despite these two doses, the infection had fulminant progression and a clinical tableau of meningoencephalitis was observed with widespread ischemic areas including bilateral basal ganglions, dentate nucleus, external capsules, medial meniscus, corpus callosum splenium and temporal lobes.

Cerebral infarction typically seems to develop within the first few days of the disease with the inflammation of the CNS. ${ }^{13}$ The mechanisms leading to cerebrovascular complications in bacterial meningitis are not completely understood and likely are multifactorial 
Invasion of the exudate into the large vessels at the base of the subarachnoid inflammatory brain may play a role. In addition, diverse bacterial components are powerful inducers of proinflammatory cytokines, possibly leading to vasculitis. ${ }^{11}$ In cases of meningoencephalitis, local inflammation of the meninges extending to the intracranial blood vessels results in ischemia and subsequent stroke.

Radiologic follow-up and outcomes of meningoencephalitis are poorly documented. In the early course of successfully-treated meningitis, CT scans of the brain are usually normal. Only a few reports about MRI findings in acute S. Pneumoniae meningitis or meningoencephalitis have been published. ${ }^{5,6}$ Another case reported was a 7-month-old girl. She presented to the hospital with seizures and was unconscious. The first CT scan was normal; however, with no improvement in neurologic status, MRI was taken and pathologic findings were observed. Peculiar, widespread and unique signal abnormalities were found on MRI in this child with meningoencephalitis caused by S. Pneumoniae with extensive central nervous white matter injury as well as evidence of thrombosis of the lateral transverse sinus. This was the first pediatric case of diffuse white matter lesions in the early course of disease in $S$. Pneumoniae meningoencephalitis. ${ }^{5}$

Infarctions in basal ganglions develop mostly secondary to chronic infections such as tuberculosis and fungal meningitis. ${ }^{7}$ Infarction in basal ganglions has rarely been described in cases of meningoencephalitis associated with S.pneumoniae infection. The only pediatric case reported in the literature was a 4-month old infant. ${ }^{7}$ The other three described cases are adults. ${ }^{14-16}$ The clinical course of the 4-monthold infant was fulminant and necrosis was seen in bilateral basal ganglions in the autopsy performed after death. ${ }^{7}$ There was basal ganglion involvement in one case reported in the literature and white matter involvement in another. However, in the present case, widespread involvement was observed in both regions.
It was reported that vaccine failure is rare. In a study of 161 pediatric cases in the United Kingdom, the vaccine failure rate was found as 0.66/100,000 in those vaccinated with PCV13. ${ }^{17}$

The causes of vaccination failure can be related to the patient, the vaccine or vaccination methods. Causes related to the patient include immune failure, patient age, eating disorder, whether the patient was healthy during vaccination or to the vaccination response in the individual. Causes related to the vaccine include the vaccine not containing some serotypes or genotypes and antigenic interaction and production-stage related reasons. Causes related to vaccination method include mistakes made during vaccine administration, not conforming to cold chain conditions during storage and transportation, and failure to follow the vaccination schedule. ${ }^{18}$

There are cases of infection reported despite vaccination. Basaranoglu et al. $^{19}$ reported infection with $19 \mathrm{~F}$ strains despite vaccination in two patients. Both patients had underlying neurologic deficits. One of the patients had a pneumonia infection, and the other had meningitis. ${ }^{19}$ Park et al. ${ }^{20}$ found an infection rate of $21 \%$ despite vaccination. The serotypes most commonly causing infection were $6 \mathrm{~B}$ and $19 \mathrm{~F} .^{20}$ Vaccine breakthrough was reported to comprise $3 \%$ of meningitis cases in France..$^{21}$ The rate of infection even with vaccination was found as $25 \%$ from 2008-2014 in Turkey. ${ }^{22}$ Again, in France, meningitis infection with 19F serotype was reported in an immunocompetent fully vaccinated 3-year-old child. ${ }^{23}$ Our patient was aged 6 months and had had two vaccination doses administered. The patient had no identified underlying immune deficiency.

To our knowledge, the present case is the first pediatric case report in the literature of involvement of both diffuse white matter and basal ganglions associated with S.pneumoniae meningoencephalitis. Moreover, the disease had fulminant progression despite two doses of PCV13. 


\section{REFERENCES}

1. Bridy-Pappas AE, Margolis MB, Center KJ, Isaacman DJ. Streptococcus pneumoniae: description of the pathogen, disease epidemiology, treatment, and prevention. Pharmacotherapy 2005; 25: 1193-1212.

2. Whitney CG, Farley MM, Hadler J, et al; Active Bacterial Core Surveillance of the Emerging Infections Program Network. Decline in invasive pneumococcal disease after the introduction of protein-polysaccharide conjugate vaccine. $\mathrm{N}$ Engl J Med 2003; 348: 1737-1746.

3. Grijalva CG, Nuorti JP, Arbogast PG, Martin SW, Edwards KM, Griffin MR. Decline in pneumonia admissions after routine childhood immunisation with pneumococcal conjugate vaccine in the USA: a time-series analysis. Lancet 2007; 369: 1179-1186.

4. Kang SJ, Kim HY, Kim YS, Lee HN, Kim HT, Kim $\mathrm{SH}$. Intractable pneumococcal meningoencephalitis associated with a TNF- $\alpha$ antagonist. J Neurol Sci 2014; 344: 215-218.

5. Jorens PG, Parizel PM, Wojciechowski $M$, et al. Streptococcus pneumoniae meningoencephalitis with unusual and widespread white matter lesions. Eur J Paediatr Neurol 2008; 12: 127-132.

6. Jorens PG, Parizel PM, Demey HE, et al. Meningoencephalitis caused by Streptococcus pneumoniae: a diagnostic and therapeutic challenge. Diagnosis with diffusion-weighted MRI leading to treatment with corticosteroids. Neuroradiology 2005; 47: 758-764

7. Magnus J, Parizel PM, Ceulemans B, Cras P, Luijks M, Jorens PG. Streptococcus pneumoniae meningoencephalitis with bilateral basal ganglia necrosis: an unusual complication due to vasculitis. J Child Neurol 2011; 26: 1438-1443.

8. Jit M. The risk of sequelae due to pneumococcal meningitis in high-income countries: a systematic review and meta-analysis. J Infect 2010; 61: 114-124.

9. Katchanov J, Heuschmann PU, Endres M, Weber JR. Cerebral infarction in bacterial meningitis: predictive factors and outcome. J Neurol 2010; 257: 716-720.

10. Snyder RD, Stovring J, Cushing AH, Davis LE, Hardy TL. Cerebral infarction in childhood bacterial meningitis. J Neurol Neurosurg Psychiatry 1981; 44: 581-585.

11. Tibussek D, Sinclair A, Yau I, et al. Late-onset group B streptococcal meningitis has cerebrovascular complications. J Pediatr 2015; 166: 1187-1192.e1.
12. Ceyhan M, Ozsurekci Y, Gurler N, et al. Distribution of Streptococcus pneumoniae serotypes that cause parapneumonic empyema in Turkey. Clin Vaccine Immunol 2013; 20: 972-976.

13. Wittebole X, Duprez T, Hantson P. Delayed cerebral ischaemic injury following apparent recovery from Streptococcus pneumoniae meningitis. Acta Clin Belg 2016; 71: 343-346.

14. Vernino S, Wijdicks EF, McGough PF. Coma in fulminant pneumococcal meningitis: new MRI observations. Neurology 1998; 51: 1200-1202.

15. Johkura K, Nishiyama T, Kuroiwa Y. Bilateral basal ganglia infarctions in a patient with Streptococcus pneumoniae meningitis. Eur Neurol 2002; 48: 123124.

16. Pugin D, Copin JC, Goodyear MC, Landis T, Gasche Y. Persisting vasculitis after pneumococcal meningitis. Neurocrit Care 2006; 4: 237-240.

17. Oligbu G, Collins S, Andrews N, et al. Characteristics and serotype distribution of childhood cases of invasive pneumococcal disease following pneumococcal conjugate vaccination in England and Wales, 2006-2014. Clin Infect Dis 2017; 65: 1191-1198.

18. Heininger U, Bachtiar NS, Bahri $\mathrm{P}$, et al. The concept of vaccination failure. Vaccine 2012; 30: 1265-1268.

19. Tanir Basaranoglu S, Karadag Oncel E, Aykac K, et al. Invasive pneumococcal disease: from a tertiary care hospital in the post-vaccine era. Human Vaccin Immunother 2017; 13: 962-964.

20. Park SY, Van Beneden CA, Pilishvili T, Martin M, Facklam RR, Whitney CG; Active Bacterial Core surveillance team. Invasive pneumococcal infections among vaccinated children in the United States. J Pediatr 2010; 156: 478-483.e2.

21. Godot C, Levy C, Varon E, Picard C, Madhi F, Cohen $R$. Pneumococcal meningitis vaccine breakthroughs and failures after routine 7-valent and 13-valent pneumococcal conjugate vaccination in children in France. Pediatr Infect Dis J 2015; 34: e260-e263.

22. Ceyhan M, Ozsurekci Y, Gurler N, et al. Serotype distribution of Streptococcus pneumoniae in children with invasive diseases in turkey: 2008-2014. Hum Vaccin Immunother 2016; 12: 308-313.

23. Isapof A, Delacourt C, Reinert P, Cohen R, Madhi F. Serotype $19 \mathrm{~F}$ pneumococcal meningitis in an imunocompetent infant immunized with heptavalent pneumococcal conjugated vaccine prevenar. Arch Pediatr 2009; 16: 1133-1136. 